\title{
Utility of microdialysis to detect the lactate/pyruvate ratio in subcutaneous tissue for the reliable monitoring of hemorrhagic shock
}

\author{
Hitoshi OHASHI ${ }^{1}$, Naruo KAWASAKI ${ }^{1}$, Shigeki FuJITANI ${ }^{1}$, \\ Katsutoshi KOBAYASHI ${ }^{2}$, Maiko OHASHI ${ }^{3}$, Akiko HOSOYAMA ${ }^{1}$, \\ Takafumi WADA ${ }^{1}$ and Yasuhiko TAIRA ${ }^{1}$ \\ ${ }^{1}$ Department of Emergency and Critical Care Medicine, St. Marianna University School \\ of Medicine, Japan \\ ${ }^{2}$ Department of Surgery, Jikei University School of Medicine, Japan \\ ${ }^{3}$ Department of Plastic Surgery, St. Marianna University School of Medicine, Japan
}

Received July 1, 2009; Accepted September 14, 2009

\begin{abstract}
The present experiments were carried out to investigate the usefulness of measuring peripheral tissue metabolism for the clinical assessment of shock. Male Wistar/ST rats (8 weeks-old) were used. All rats were placed in a supine position while anesthetized. A tube for measuring arterial pressure and collecting blood samples was cannulated into the femoral artery. For microdialysis, the introducer was inserted into the subcutaneous tissue in the abdominal wall. Blood was exsanguinated to maintain the mean arterial pressure at $40 \pm 5 \mathrm{mmHg}$. Mean arterial pressure, arterial blood gas and serum lactate levels were measured. Microdialysis was performed to quantify the levels of lactate and pyruvate in the subcutaneous tissue. Six rats died due to hemorrhagic shock by 350 min (Group D) while six rats had survived for the $350 \mathrm{~min}$ period after exsanguination (Group A). These data was obtained at intervals of $50 \mathrm{~min}$ after exsanguination up to a period of $250 \mathrm{~min}$ and compared between Groups A and D. In Group A, serum lactate levels did not increase throughout the entire period of observation. Serum lactate levels in Group D transiently increased, but did not show a dramatic increase during the blood pressure maintenance period. In particular, serum lactate levels increased again after a period of more than 150 min following exsanguination. Lactate levels in the subcutaneous tissue gradually increased and were significantly higher in Group D than that in Group A after 150 min. The $\mathrm{L} / \mathrm{P}$ ratio in Group A remained fairly constant during the period of observation. In contrast, the $\mathrm{L} / \mathrm{P}$ ratio in Group $\mathrm{D}$ increased gradually, and was significantly higher than that in Group A after 100 min. It was concluded that the continuous increase in the $\mathrm{L} / \mathrm{P}$ ratio in the subcutaneous tissue in Group D was indicative of tissue circulatory failure and of an abnormality in tissue oxygen metabolism prior to the detection of the collapse of compensatory mechanisms appearing in the vital signs. These findings suggest that measuring the $\mathrm{L} / \mathrm{P}$ ratio is useful for the clinical assessment and monitoring of shock.
\end{abstract}

Key words: microdialysis, lactate, lactate/pyruvate ratio, subcutaneous tissue, hemorrhagic shock 


\section{Introduction}

Mortality rates secondary to shock caused by trauma, surgery or sepsis and subsequent multiple organ failure remains high (Fink et al., 1991; Chieveley-Williams et al., 1999). Shock was previously defined as a state of markedly impaired peripheral circulation due to decreased blood pressure. More recently, shock has been defined as a state in which blood flow to important organs cannot be maintained, resulting in cytological tissue metabolism disorder and organ damage (Kruse, 1999). Cytological tissue metabolism disorder and organ damage are caused by hypoxemia, low tissue perfusion, reduced tissue oxygen usage and dysoxia (Robin, 1977).

The condition in which metabolic energy production is limited by the supply or utilization of oxygen is called dysoxia, with the clinical expression of this condition known as shock (Connett et al., 1990). Dysoxia can be the result of an inadequate supply of oxygen, as occurs in hypovolemic shock and cardiogenic shock, or it can be caused by a defect in mitochondrial oxygen utilization, as occurs in septic shock.

Severity assessment and the early detection of shock due to peripheral circulatory failure are thus important for prognosis. APACHE II and III are often used to assess the severity of shock and prognosis (Cerra et al., 1990; Knaus et al., 1991). APACHE II is highly reliable, but is likely to be affected by metabolism and treatment, and apparent improvements can hinder the accurate assessment of the severity.

Serum lactate levels are easily measured, and have been frequently quantified to ascertain systemic tissue metabolism (Bernardin et al., 1996; Vitek et al., 1971). Serum lactate levels are affected by the rate of production as well as by the metabolism of the whole body and therefore do not precisely indicate the metabolic activity of local tissue.

Then, Ungerstedt (1991) measured lactate in subcutaneous tissue and the peritoneum by microdialysis (MD) and reported that anaerobic metabolism could be detected much earlier by this method. In the event of a tissue metabolism disorder, blood distribution imbalance occurs to protect important organs. Therefore the blood flow to subcutaneous tissue, muscle and the peritoneum decreases. MD enables the quantification of changes in the elevation of the tissue lactate levels and of the lactate/pyruvate $(\mathrm{L} / \mathrm{P})$ ratio.

In fact, during intensive care management of high-risk infants, MD of subcutaneous tissue has been useful in the measurement of metabolic products without repeated collection of blood samples, with adverse reactions reportedly tolerable even when an MD device was placed subcutaneously for 1-16 days (Hack et al., 2005).

Hence, lactate levels and the $\mathrm{L} / \mathrm{P}$ ratio in subcutaneous tissue precisely reflects the shock status in patients. However, few studies have compared tissue lactate levels to conventional indicators such as systemic arterial pressure, blood gas, hematocrit (Ht) and serum lactate, and the reliability of these needs to be assessed.

In this study we have used animal models of hemorrhagic shock and investigated the usefulness of measuring peripheral tissue metabolism for the clinical assessment of shock. 


\section{Methods}

\section{Experimental animal preparation}

Male Wistar/ST rats (8 weeks old, 70-330 g, Japan SLC Inc, Shizuoka, Japan) were used. After 6 hours of fasting, $3 \mathrm{ml}$ of diethyl ether was used to induce anesthesia. Anesthesia was maintained by administering $2.0 \mathrm{l} / \mathrm{min}$ of $1.2 \%$ isoflurane using a systemic anesthetic apparatus for laboratory animals. All animals were placed in a supine position and managed with spontaneous respiration.

A tube (inner diameter, $0.5 \mathrm{~mm}$; outer diameter, $0.8 \mathrm{~mm}$; SP31: Natsume Seisakusho Co. Ltd., Osaka, Japan) for measuring mean arterial pressure (MAP) and collecting blood samples was cannulated into the left femoral artery. MAP was measured using a blood pressure transducer (Lifekit DX-360; Becton, Dickinson and Company, Tokyo Japan) via the catheter tube.

\section{Preliminary study}

A control study was conducted using Wistar/ST rats $(\mathrm{n}=3)$. To simulate hemorrhagic shock $(\mathrm{n}=3)$, blood was slowly removed for $50 \mathrm{~min}$ to maintain MAP at $40 \pm 5 \mathrm{mmHg}$. As indicators of peripheral tissue metabolism, MD was performed to measure both lactate and pyruvate levels in the interstitial tissue of their abdominal subcutaneous tissue. MAP, arterial blood gas and serum lactate levels were measured simultaneously. This preliminary study showed that lactate levels in both subcutaneous tissue and serum increased.

\section{Study design}

After confirming systemic circulatory stability, blood was exanguinated for $50 \mathrm{~min}$ to maintain MAP at $40 \pm 5 \mathrm{mmHg}$. Six rats had died due to hemorrhagic shock within a $350 \mathrm{~min}$ period following exanguination (Group $D ; n=6$ ) while 6 rats had survived for the same period following exsanguination (Group $A ; n=6$ ). Data were compared between these two groups for the 250 min period following exsanguination. Mean volumes of exsanguination were $10.1 \pm 0.9$ $\mathrm{ml}$ in Group D and $11.4 \pm 0.8 \mathrm{ml}$ in Group A.

\section{Measurement methods and items}

Using the catheter in the femoral artery, $100 \mu \mathrm{l}$ of blood was collected at $0,50,100,150,200$ and 250 min following exsanguination. Arterial blood gas and serum lactate were measured by i-STAT (i-STAT Corporation, Illinois, U.S.A.). MAP was measured at the same time.

\section{Microdialysis}

MD was performed to quantify lactate and pyruvate levels in the interstitial tissue of the abdominal subcutaneous tissue. The introducer (CMA/20 14/10 PC probe, CMA Microdialysis $\mathrm{AB}$, Solna, Sweden) was inserted into the subcutaneous tissue in the middle abdominal wall. At the end of the study, the skin was dissected to ensure that the probe had been properly placed in subcutaneous tissue. A perfusion solution of lactate free Ringer's solution $\left(\mathrm{Na}^{+} 147 \mathrm{mEq} / 1, \mathrm{~K}^{+}\right.$ $\left.4 \mathrm{mEq} / \mathrm{l}, \mathrm{Ca}^{2+} 5 \mathrm{mEq} / \mathrm{l}, \mathrm{Cl}^{-} 156 \mathrm{mEq} / \mathrm{l}\right)$ was injected into the tissue at a rate of $1.0 \mu \mathrm{l} / \mathrm{min}$ using 
a syringe pump (CMA400, CMA Microdialysis AB, Solna, Sweden). Equilibration of substances in the interstitial and perfusion fluid takes place by diffusion along concentration gradients. The dialysate, which streams back in the inner lumen, reflects the interstitial concentration of metabolites. Microdialysis values give a valid and direct estimate of tissue metabolite concentrations, and allow semi-continuous biochemical monitoring of cellular metabolism without repeated puncture trauma of the tissue (Klaus et al., 2003).

In this research, the lactate and pyruvate levels in the dialysate were measured at 0, 50, 100, 150, 200 and 250 min following exsanguination. The dialysate was analyzed spectrophotometrically for concentrations of lactate and pyruvate by the Microdialysis analyzer (CMA600, CMA Microdialysis AB, Solna, Sweden).

\section{Statistical analysis}

The results were expressed as the mean \pm standard error of the mean (SEM), and the Mann-Whitney's U-test was used to evaluate the results. Statistical significance was set at $P<0.05$ (Stat View 5.0; SAS Institute Inc. USA).

\section{Experimental guideline}

The above animal study was conducted in accordance with the Animal Study Guidelines of the Ethical Committee of St Marianna University School of Medicine.

\section{Results}

\section{Amount of exsanguination}

No significant changes were seen in mean volumes of exsanguination in either group (Group D, $10.1 \pm 0.9 \mathrm{ml}$; Group A, $11.4 \pm 0.8 \mathrm{ml}$ ).

\section{MAP and arterial blood gas}

Figure 1 and Table 1 show chronological changes in MAP, Ht and arterial blood gas levels in Groups A and D. Immediately after exsanguination, no significant changes were seen in MAP or arterial blood gas levels in either group. In both groups, blood pressure transiently recovered after the shock induced by hemorrhage. There was no significant difference in the MAP between the two groups until after 150 min following exsanguination when the Group D MAP significantly decreased $(P<0.05)$.

Arterial blood gas analysis showed that the $\mathrm{Ht}$ at $250 \mathrm{~min}$ after exsanguination was significantly higher in Group D than that of Group A $(P<0.05)$. In Group D, the pH decreased gradually over time, but the $\mathrm{pH}$ in Group A remained constant at $7.3 \pm 0.0(P<0.05)$. In Group A, $\mathrm{PaCO}_{2}$ at both 50 and $100 \mathrm{~min}$ after exsanguination was high $(P<0.05)$, but no significant differences were seen thereafter. In Group $\mathrm{D}, \mathrm{PaO}_{2}$ was significantly higher immediately after exsanguination $(P<0.05$ or 0.01$)$. $\mathrm{HCO}_{3}{ }^{-}$significantly decreased after a period of $100 \mathrm{~min}$ following exsanguination in Group D $(P<0.05)$. 


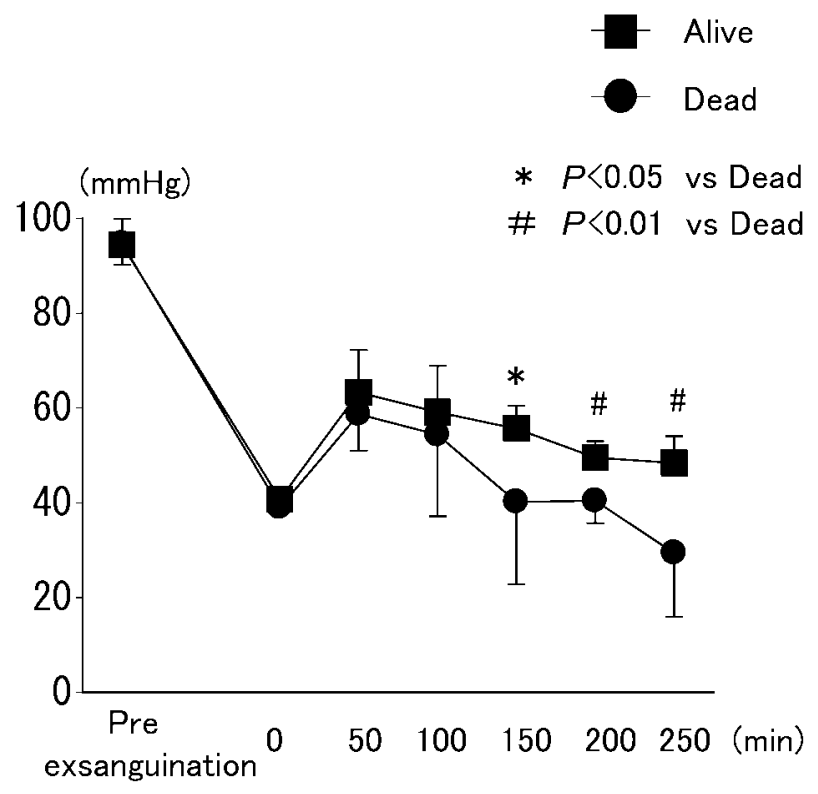

Fig. 1. Mean arterial pressure (MAP). Immediately after exsanguination, no significant changes were seen in MAP. In both groups, blood pressure transiently recovered after the shock induced by hemorrhage. In Group D, MAP significantly decreased after a period of $150 \mathrm{~min}$ following exsanguination $(P<0.05)$.

Table 1 Arterial blood gas analysis showed that the Ht at $250 \mathrm{~min}$ following exsanguination was significantly higher in Group D than in Group A $(P<0.05)$

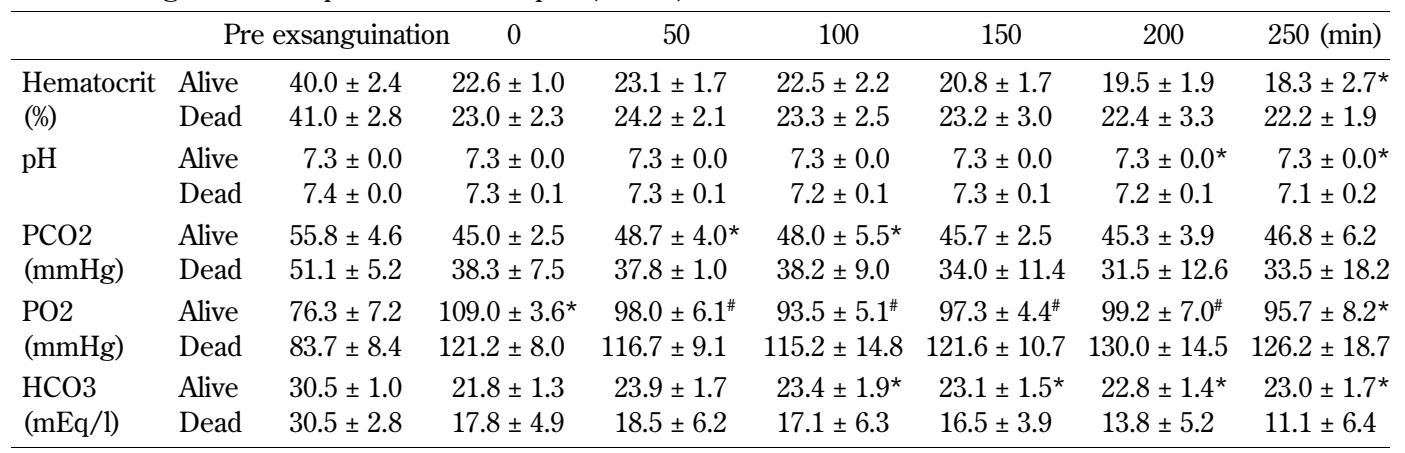

In Group $\mathrm{D}$, $\mathrm{pH}$ decreased over time $(P<0.05)$. In Group $\mathrm{A}, \mathrm{PaCO} 2$ at 50 and $100 \mathrm{~min}$ following exsanguination was higher $(P<0.05)$, but no significant differences were seen thereafter. In Group $\mathrm{D}, \mathrm{PaO} 2$ was significantly higher immediately after exsanguination $(P<0.05$ or 0.01$)$. $\mathrm{HCO}_{3}{ }^{-}$significantly decreased after a period of $50 \mathrm{~min}$ following exsanguination in Group D, but remained stable in Group A $(P<0.05)$. ${ }^{*}, P<0.05$ vs. Dead;,$P<0.01$ vs. Dead.

\section{Comparison of lactate levels in serum and subcutaneous tissue}

Figure 2 shows chronological changes in the lactate levels of serum and in the subcutaneous tissue. At exsanguination ( $0 \mathrm{~min})$, no significant difference in serum lactate was seen between these 2 groups. In Group A, serum lactate did not increase throughout the entire period of observation. Serum lactate in Group D is higher than that of Group A after a period of 
lactate in serum

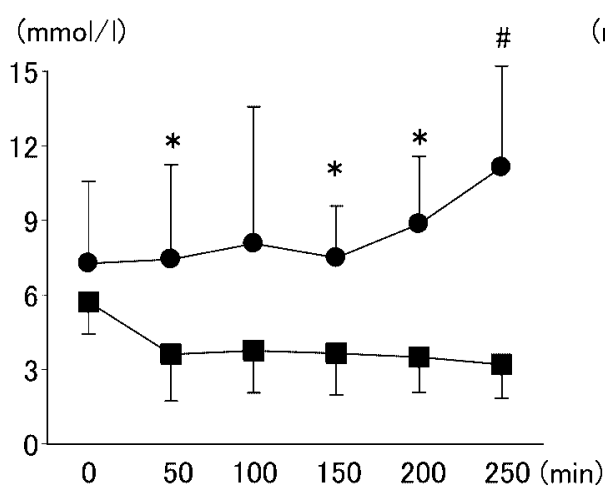

lactate in subcutaneous tissue

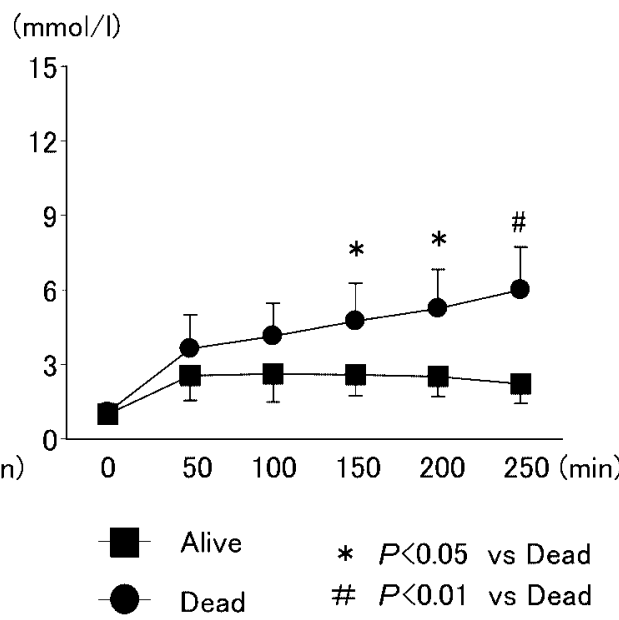

Fig. 2. Comparison of lactate levels in serum and subcutaneous tissue. Serum lactate levels in Group D significantly increased after a period of $50 \mathrm{~min}$ following exsanguination $(P<0.05)$. In particular, after a period of $250 \mathrm{~min}$ following exsanguination, the increase in serum lactate levels was associated with a decline in blood pressure $(P<0.01)$. In Group A, serum lactate levels did not increase during the blood pressure maintenance period. The lactate level in the subcutaneous tissue in Group D was significantly higher than that in Group A after a period of $150 \mathrm{~min}$ following exsanguination $(P<0.05$ or 0.01 ).

50 min following exsanguination $(P<0.05)$. But serum lactate levels did not show a dramatic increase during the blood pressure maintenance period. In particular, serum lactate levels increased again after a period of 150 min following exsanguination.

Lactate levels in the subcutaneous tissue gradually increased following exsanguination. Lactate levels in subcutaneous tissue in Group D were significantly higher than that in Group A after a period of $150 \mathrm{~min}$ following exsanguination $(P<0.05$ or 0.01$)$.

\section{$L / P$ ratio in subcutaneous tissue}

Figure 3 shows the chronological changes in the subcutaneous tissue L/P ratio in Groups $\mathrm{A}$ and $\mathrm{D}$. The $\mathrm{L} / \mathrm{P}$ ratio in Group A remained fairly constant during the period of observation. In contrast, the $\mathrm{L} / \mathrm{P}$ ratio in Group $\mathrm{D}$ increased gradually, and was significantly higher than that in Group A after $100 \min (P<0.05$ or 0.01$)$.

\section{Discussion}

Hypotension is not required to define shock, with a greater importance being assigned to the presence of inadequate tissue perfusion on physical examination. Given the current evidence, the only bio-marker recommended for diagnosis or staging of shock is blood lactate levels (Antonelli, 2007). Shock is defined as an inadequate tissue perfusion and tissue oxygen metabolism abnormality. 


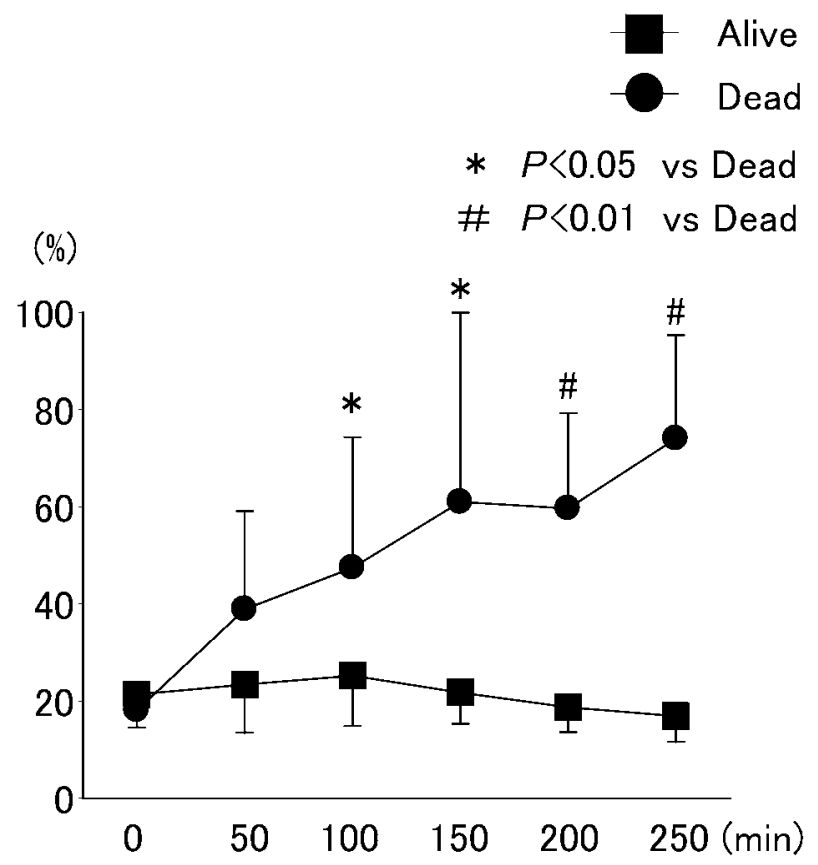

Fig. 3. $\mathrm{L} / \mathrm{P}$ ratio in subcutaneous tissue. In Group $\mathrm{D}$, the $\mathrm{L} / \mathrm{P}$ ratio significantly increased during the period of $100-250$ min following exsanguination $(P<0.05$ or 0.01$)$. Conversely, the $\mathrm{L} / \mathrm{P}$ ratio remained constant without marked fluctuation in Group $\mathrm{A}$.

Our results suggest that monitoring the $\mathrm{L} / \mathrm{P}$ ratio and lactate levels in subcutaneous tissue by $\mathrm{MD}$, even during a period of stable blood pressure, allows the early detection of shock.

With regard to arterial pressure, even if hemorrhagic shock lowers cardiac output, apparent homeostasis is achieved with transient circulatory compensation. However, severe hemorrhagic shock disturbs such compensatory mechanisms, and poor hemodynamics and microcirculatory failure cause peripheral tissue dysfunction. Detecting hemorrhagic shock in peripheral tissues at an early stage and then properly treating the dysfunction prior to the collapse of the compensatory mechanisms should improve the prognosis.

In the present study, blood pressure transiently recovered during the early stage following exsanguination, but decreased over time. This was because hemorrhage resulted in the sympathetic nervous system releasing catecholamines and stimulated the renin-angiotensin system, thus elevating systemic vascular resistance, increasing heart rate and transiently increasing blood pressure in a compensatory manner. Over time, because of diminution of the compensatory mechanisms, it becomes more difficult to maintain a stable blood pressure. No marked differences in blood pressure existed between Group D and A up to $100 \mathrm{~min}$ following exsanguination, which suggests that shock is difficult to assess based solely on blood pressure. Similarly, arterial blood gas analysis showed apparent improvements in $\mathrm{PaCO}_{2}$ and $\mathrm{PaO}_{2}$ in Group D. This appears to be evidence of the strong compensatory mechanisms that activate with shock, and suggests that both $\mathrm{PaCO}_{2}$ and $\mathrm{PaO}_{2}$ are unsuitable for the clinical assessment of shock. 
We used microdialysis to measure tissue lactate levels in our study. MD is a unique technique for monitoring the chemistry of the extracellular space in living tissue. Ungerstedt $e t$ al. (2004) first used narrow artificial vessels in artificial dialysis to successfully recover monoamines in the brain. Microdialysis works by slowly pumping a solution through the microdialysis probe. By embedding a dialysis tube with a diameter of $0.2-0.5 \mathrm{~mm}$ into the body, various compounds in the intercellular space can be collected in the irrigation fluid as a result of simple diffusion down concentration gradients (Klaus et al., 2004). The most successful area of MD use is in the neuroscience field. During neurointensive care, MD is used to evaluate how seriously brain cells are affected by ischemia, trauma, and hemorrhage. Sampling has been used to study changes in the levels of lactate, glycerol and glutamate. MD works in cytological tissue, and thus it can be used as a predictive indicator of cellular change in advance of a blood examination.

In basic research, MD has been used to assess musculocutaneous flaps (Setälä et al., 2007), while in clinical research it has allowed monitoring of local metabolic changes in the skin that cannot be ascertained by circulatory dynamic analysis in patients with extensive burns (Samuelsson et al., 2006). When MD was performed in the peritoneum of patients who underwent gastrointestinal surgery, the L/P ratio, as an indicator of anaerobic metabolism, correlated with the levels of inflammatory cytokines, Tumor Necrosis Factor-alpha (TNF- $\alpha$ ) and Interleukin-10 (IL-10) (Jansson et al., 2004).

Visceral ischemia is an important factor in multiple organ failure, and lactate levels and the $\mathrm{L} / \mathrm{P}$ ratio are well-known markers for anaerobic metabolism and ischemia. Ischemia is defined as a decrease in blood flow causing an inadequate supply of both oxygen and glucose. The cell takes up as much glucose as possible in order to produce ATP by anaerobic glycolysis. The decrease in glucose delivery from the capillaries together with an increase in glucose uptake leads to a fall in the glucose concentration in the dialysate. More lactate is produced in order to regenerate nicotinamide adenine dinucleotide (NAD) ${ }^{+}$which is necessary for maintaining anaerobic glycolysis. The result will be an increase in lactate levels and a decrease in pyruvate levels and hence an increase in the lactate/pyruvate ratio.

Serum lactate levels can be measured simultaneously with blood gases, and thus provide a convenient indicator for detecting tissue oxygen metabolism abnormality. In the present study, serum lactate levels were significantly higher in Group D than in Group A, suggesting that monitoring serum lactate levels could be useful in the diagnosis of severe hemorrhagic shock. However, serum lactate levels are a balance between tissue production and its metabolism by organs such as the liver, heart and brain. Therefore, when monitoring serum lactate levels during a blood pressure maintenance period, serum lactate is affected by metabolism, so that the lack of apparent change in levels makes serum lactate unsuitable for the clinical assessment and monitoring of shock.

The results in Group D were scrutinized further. Irrespective of blood pressure changes due to reduced blood circulation, lactate levels and the $\mathrm{L} / \mathrm{P}$ ratio in subcutaneous tissue increased continuously. Lactate levels in subcutaneous tissue showed a steady increase after a period of 150 min following exsanguination. And the L/P ratio increased from $100 \mathrm{~min}$ after exsanguination in our study. Lactate levels, and particularly the L/P ratio in subcutaneous 
tissue, continuously increased. Thus this result allows the detection of tissue circulatory failure prior to the collapse of the compensatory mechanisms that mask this in vital signs. The $\mathrm{L} / \mathrm{P}$ ratio may be useful for the early detection of shock.

Measurement of the $\mathrm{L} / \mathrm{P}$ ratio with $\mathrm{MD}$ has been used in the human peritoneum following laparotomy for mesenteric ischemia (Ungerstedt et al., 2003) and right colectomy (Jansson et $a l ., 2003)$. It increased before the onset of hypoxia or arterial thrombosis. However, L/P ratio measurement in the peritoneum is highly invasive, and cannot be routinely performed in critically ill patients. Conversely, measurement of the $\mathrm{L} / \mathrm{P}$ ratio in subcutaneous tissue is less invasive. These findings suggest that measuring $\mathrm{L} / \mathrm{P}$ ratio in subcutaneous tissue is useful for the clinical assessment and monitoring of shock.

Our results suggest that measurement of the $\mathrm{L} / \mathrm{P}$ ratio in subcutaneous tissue using the $\mathrm{MD}$ method is more sensitive and a possible early marker for the detection of hemorrhagic shock. Further study is needed in patients with hemorrhagic shock.

\section{References}

Antonelli, M., Levy, M., Andrews, P.J., Chastre, J., Hudson, L.D., Manthous, C., Meduri, G.U., Moreno, R.P., Putensen, C., Stewart, T. and Torres, A. (2007). Hemodynamic monitoring in shock and implications for management. Intensive Care Med. 33: 575-590.

Bernardin, G., Pradier, C., Tiger, F., Deloffre, P. and Mattei, M. (1996). Blood pressure and arterial lactate level are early indicators of short-term survival in human septic shock. Intensive Care Med. 22: 17-25.

Cerra, F.B., Negro, F. and Abrams, J. (1990). APACHE II score does not predict multiple organ failure or mortality in postoperative surgical patients. Arch. Surg. 125: 519-522.

Chieveley-Williams, S. and Hamilton-Davies, C. (1999). The role of the gut in major surgical postoperative morbidity. Int. Anesthesiol. Clin. 37: 81-110.

Connett, R.J., Honig, C.R., Gayeski, T.E. and Brooks, G.A. (1990). Defining hypoxia: a systems view of VO2, glycolysis, energetics, and intracellular PO2. J. Appl. Physiol. 68: 833-842.

Fink, M.P. (1991). Gastrointestinal mucosal injury in experimental models of shock, trauma, and sepsis. Crit. Care Med. 19: 627-641.

Hack, A., Busch, V., Gempel, K. and Baumeister, F.A. (2005). Subcutaneous microdialysis for childrensafe biochemical tissue monitoring based on a minimal traumatizing no touch insertion technique. Eur. J. Med. Res. 10: 419-425.

Jansson, K., Ungerstedt, J., Jonsson, T., Redler, B., Andersson, M., Ungerstedt, U. and Norgren, L. (2003). Human intraperitoneal microdialysis: increased lactate/pyruvate ratio suggests early visceral ischemia, a pilot study. Scand. J. Gastroenterol. 38: 1007-1011.

Jansson, K., Redler, B., Truedsson, L., Magnuson, A., Ungerstedt, U. and Norgren, L. (2004). Postoperative on-line monitoring with intraperitoneal microdialysis is a sensitive clinical method for measuring increased anaerobic metabolism that correlates to the cytokine response. Scand. J. Gastroenterol. 39: 434-439.

Klaus, S., Heringlake, M. and Bahlmann, L. (2004). Bench-to-bedside review: microdialysis in intensive care medicine. Crit. Care. 8: 363-368.

Klaus, S., Heringlake, M., Block, K., Nolde, J., Staubach, K. and Bahlmann, L. (2003). Metabolic changes detected by microdialysis during endotoxin shock and after endotoxin preconditioning. Intensive Care Med. 29: 634-641.

Kruse, J.A. (1999). Searching for the perfect indicator of dysoxia. Crit. Care Med. 27: 469-471. 
Knaus, W.A., Wagner, D.P., Draper, E.A., Zimmerman, J.E., Bergner, M., Bastos, P.G., Sirio, C.A., Murphy, D.J., Lotring, T. and Damiano, A. (1991). The APACHE III prognostic system. Risk prediction of hospital mortality for critically ill hospitalized adults. Chest 100: 1619-1636.

Robin, E.D. (1977). Dysoxia: abnormal tissue oxygen utilization. Arch. Intern. Med. 137: 905-910.

Samuelsson, A., Steinvall, I. and Sjöberg, F. (2006). Microdialysis shows metabolic effects in skin during fluid resuscitation in burn-injured patients. Crit. Care 10: R172.

Setälä, L., Joukainen, S., Uusaro, A., Alhava, E. and Härmä, M. (2007). Metabolic response in microvascular flaps during partial pedicle obstruction and hypovolemic shock. J. Reconstr. Microsurg. 23: 489-496.

Ungerstedt, U. (1991). Microdialysis-principles and applications for studies in animals and man. $J$. Intern. Med. 230: 365-373.

Ungerstedt, J., Nowak, G., Ericzon, B.G. and Ungerstedt, U. (2003). Intraperitoneal microdialysis (IPM): a new technique for monitoring intestinal ischemia studied in a porcine model. Shock 20: 91-96.

Ungerstedt, U. and Rostami, E. (2004). Microdialysis in neurointensive care. Curr. Pharm. Des. 10: 2145-2152.

Vitek, V. and Cowley, R.A. (1971). Blood lactate in the prognosis of various forms of shock. Ann. Surg. 173: 308-313. 